\title{
Electroacupuncture at Bilateral Zusanli Points (ST36) Protects Intestinal Mucosal Immune Barrier in Sepsis
}

\author{
Mei-fei Zhu, Xi Xing, Shu Lei, Jian-nong Wu, Ling-cong Wang, \\ Li-quan Huang, and Rong-lin Jiang \\ Department of Critical Care Medicine, The First Affiliated Hospital, Zhejiang Chinese Medical University, Hangzhou 310006, China \\ Correspondence should be addressed to Rong-lin Jiang; jiangronglin@126.com
}

Received 1 December 2014; Revised 20 March 2015; Accepted 10 April 2015

Academic Editor: Tetsuya Kondo

Copyright (c) 2015 Mei-fei Zhu et al. This is an open access article distributed under the Creative Commons Attribution License, which permits unrestricted use, distribution, and reproduction in any medium, provided the original work is properly cited.

\begin{abstract}
Sepsis results in high morbidity and mortality. Immunomodulation strategies could be an adjunctive therapy to treat sepsis. Acupuncture has also been used widely for many years in China to treat sepsis. However, the underlying mechanisms are not well-defined. We demonstrated here that EA preconditioning at ST36 obviously ameliorated CLP-induced intestinal injury and high permeability and reduced the mortality of CLP-induced sepsis rats. Moreover, electroacupuncture (EA) pretreatment exerted protective effects on intestinal mucosal immune barrier by increasing the concentration of sIgA and the percentage of $\mathrm{CD} 3+, \gamma / \delta$, and CD4+ T cells and the ratio of CD4+/CD8+ T cells. Although EA at ST36 treatments immediately after closing the abdomen in the CLP procedure with low-frequency or high-frequency could not reduce the mortality of CLP-induced sepsis in rats, these EA treatments could also significantly improve intestinal injury index in rats with sepsis and obviously protected intestinal mucosal immune barrier. In conclusion, our findings demonstrated that EA at ST36 could improve intestinal mucosal immune barrier in sepsis induced by CLP, while the precise mechanism underlying the effects needs to be further elucidated.
\end{abstract}

\section{Introduction}

Sepsis frequently occurs after trauma, burns, hemorrhage, or abdominal surgery. It can progress to multiorgan failure (MOF). Although new treatment algorithms focusing on rapid administration of broad spectrum antibiotics and aggressive restoration of tissue oxygen delivery have led to decreases in mortality, the death rate is still high $(\sim 30 \%)$ [1-4]. Effective and safe treatments for this disease are desperately needed.

Sepsis is a complex immune syndrome characterized by an imbalance between pro- and anti-inflammatory mediators systemically released in high amounts (cytokine storm) in response to an infection, while the precise pathophysiologic mechanisms underlying the development of multiorgan failure (MOF) remain elusive $[5,6]$. The gut is often described as the motor of MOF because the loss of its integrity is a critical comorbidity factor for patients after HS, as well as trauma, surgery, sepsis, and burn injuries $[7,8]$. Therefore, the intestinal mucosal immune system has recently emerged as a potential target in sepsis treatment.

Traditional Chinese medicine (TCM, including Chinese materia medica, acupuncture, and physiotherapy) always attached importance to gastrointestinal function, believing that the stomach and spleen provide the material basis for the acquired constitution. Acupuncture has also been used widely for many years in China to treat sepsis. Recently, acupuncture has been described as a complementary and alternative medicine (CAM) in which filiform needles are inserted at specific points on the body, called acupoints, which can subsequently be stimulated in various ways, such as electroacupuncture (EA) [9]. It has been reported that in rats with CLP sepsis models EA-ST36 reduces serum TNF levels through VN- and catecholamine-dependent mechanisms. Indeed, the treatment with EA at ST36 can decrease levels of proinflammatory cytokine expression (TNF- $\alpha$, IL1 , and IL-6) in a lipopolysaccharide-induced model of acute 
nephritis, collagen-induced arthritis mouse model, ulcerative colitis rat model, carrageenan-induced mouse model of inflammation, and cerebral ischemia-reperfusion injured rats. Recent studies have shown that the mechanism underlying the effects of this treatment is related to the suppression of the TLR4/NF- $\kappa$ B signaling pathway [10-13].

Although the recent focus has been on the function of EA at ST36, there are a lot of problems to be solved and whether EA at ST36 protects gut barrier is uncertain. The object of this study, based on the rat model of sepsis induced by CLP, was to observe the effects of EA at ST36 on the intestinal mucosal immune barrier.

\section{Materials and Methods}

2.1. Animals. Male Sprague Dawley (SD) rats (body weight, $180-220 \mathrm{~g}$ ) were obtained from the Zhejiang province Experimental Animal Center and housed in the laboratory animal center of Zhejiang Chinese Medical University at $22^{\circ} \mathrm{C}$ with a 12-hour light/dark cycle. All animals used in the study were housed and cared for in accordance with the Chinese Pharmacological Society Guidelines for Animal Use. The work was approved by the Committee on the Ethics of Animal Experiments of the Zhejiang Chinese Medical University (permit number: 2012-0049). All surgery was performed under sodium pentobarbital anesthesia, and all efforts were made to minimize suffering.

2.2. Experimental Sepsis Model by CLP. The rats were subjected to CLP as previously described. Briefly, under aseptic conditions, a $3-\mathrm{cm}$ midline laparotomy was performed to allow the exposure of the cecum and adjoining intestine. The cecum was tightly ligated with a 2.0-silk suture at its base, below the ileocecal valve, and was perforated twice with an 18-gauge needle. The cecum was then gently squeezed to extrude a small amount of feces from the puncture site. The cecum was then returned to the peritoneal cavity and the laparotomy was closed with 3.0-silk sutures. Sham-operated animals underwent the same surgical procedure although the cecum was neither ligated nor punctured. Saline $(3 \mathrm{~mL} / 100 \mathrm{~g})$ was administered to all rats intraperitoneally at the end of the procedure. All animals were returned to their cages with free access to food and water.

2.3. Acupuncture Treatment Procedure. Two pairs of stainless steel needles (diameter, $0.3 \mathrm{~mm}$; length, $30 \mathrm{~mm}$ (Suzhou Medical Supplies Co., Jiangsu, China)) were inserted perpendicularly at a depth of $6 \mathrm{~mm}$ into the bilateral Zusanli acupoints (ST36), located $5 \mathrm{~mm}$ below and lateral to the anterior tubercle of the tibia. EA stimulation was applied at both bilateral ST36 acupoints, and both output leads from the Programmable Electro-Acupuncture Stimulator (HANS, LH202H, Huawei Co., Beijing, China) were connected to the handles of both needles inserted at ST36 acupoints. EA was applied for $30 \mathrm{~min}$, with an intensity of $2 \mathrm{~mA}$ and $2-100 \mathrm{~Hz}$.

2.4. Experimental Groups and Protocol. The five groups of animals used in the present study were (1) the sham-operated group (sham, $n=20$ ), which underwent a laparotomy; (2) the sepsis group (sepsis, $n=20$ ), which underwent CLP; (3) the low-frequency EA group (sepsis + low-frequency EA, $n=20$ ), which underwent ST36 acupuncture immediately after closing the abdomen in the CLP procedure and 24 hrs later; (4) the high-frequency EA group (sepsis + high-frequency EA, $n=20$ ), which underwent ST36 acupuncture immediately after closing the abdomen in the CLP procedure and $6 \mathrm{hrs}, 12 \mathrm{hrs}, 18 \mathrm{hrs}$, and $24 \mathrm{hrs}$ later; and (5) EA preconditioning group (EA + sepsis, $n=20)$, which underwent CLP immediately prior to the application of five days of ST36 acupuncture, once a day.

The rats were kept at a constant environmental temperature of $37^{\circ} \mathrm{C}$ to maintain body heat following the procedures. At $36 \mathrm{~h}$ after the CLP, the rats were reanesthetized, then their abdomens were opened, and ileum was removed for the determination of intestinal mucosal tissue sIgA levels, flow cytometry assay, and histomorphological determination.

2.5. Histological Examination. The fixed intestinal mucosal tissue was cut into $3-\mathrm{mm}$ thickness blocks. The tissue blocks were embedded in paraffin and cut into $4 \mu \mathrm{m}$ slices. After being deparaffinized using xylene and ethanol dilutions and rehydration, the sections were stained with hematoxylin and eosin (H\&E, Bogoo, Shanghai, $\mathrm{CN}$ ) to examine the tissue structure, inflammatory cell infiltration, and necrosis.

2.6. sIgA Measurement in Intestinal Mucosa and D-Lactose Measurement in Plasma. Intestinal mucosal tissue sIgA level and D-Lactose measurement in plasma were determined by using ELISA Kit (Boster biological Inc., Wuhan, China) following the manufacturer's protocol.

2.7. Flow Cytometry Assay. Follicle-free mucosa destined for mononuclear cells (MC) isolation was cut into smaller pieces of approximately $1 \mathrm{~mm}^{2}$. These mucosa pieces were incubated separately for $30 \mathrm{~min}$ at $37^{\circ} \mathrm{C}$ in calcium- and magnesiumfree Hanks's balanced salt solution, including $1 \mathrm{mM}$ ethylenediamine tetra-acetic acid (Sigma-Aldrich, Shanghai, China) to remove both epithelium and intraepithelial lymphocytes. After washing, the follicle-free mucosal tissue was disrupted mechanically into smaller pieces of approximately $1-2 \mathrm{~mm}^{3}$ and incubated in RPMI-1640 medium supplemented with $30 \mathrm{mM}$ HEPES, $10 \%$ fetal calf serum (FCS), $100 \mathrm{U} / \mathrm{mL}$ penicillin, $100 \mu \mathrm{g} / \mathrm{mL}$ streptomycin, $50 \mu \mathrm{g} / \mathrm{mL}$ gentamicin, and $2.5 \mu \mathrm{g} / \mathrm{mL}$ amphotericin (all from MCE, Shanghai, China), $0.1 \%$ collagenase type IV (Sigma-Aldrich, Shanghai, China), 0.05\% DNase I (Roche Diagnostics, Shanghai, China), and $1 \mu \mathrm{L} / \mathrm{mL} 2$-mercaptoethanol (Sigma-Aldrich, Shanghai, China) at $37^{\circ} \mathrm{C}$ in humidified $5 \% \mathrm{CO}_{2}$ atmosphere for $1.5 \sim$ $2 \mathrm{hrs}$ with continuous agitation and vigorous vortex every $15 \mathrm{~min}$. Remaining tissue aggregates were removed by a $70 \mu \mathrm{m}$ nylon cell strainer. The resulting cell suspension was centrifuged at $500 \mathrm{~g}$ for $25 \mathrm{~min}$ in a $30 \%$ isotonic Percoll solution (Borunlaite Sci \& Tech Co., Ltd., Beijing, China). The supernatant containing epithelial cells and debris was discarded; the cell pellet was washed and resuspended in RPMI1640 medium. The MC fraction additionally underwent Ficoll 


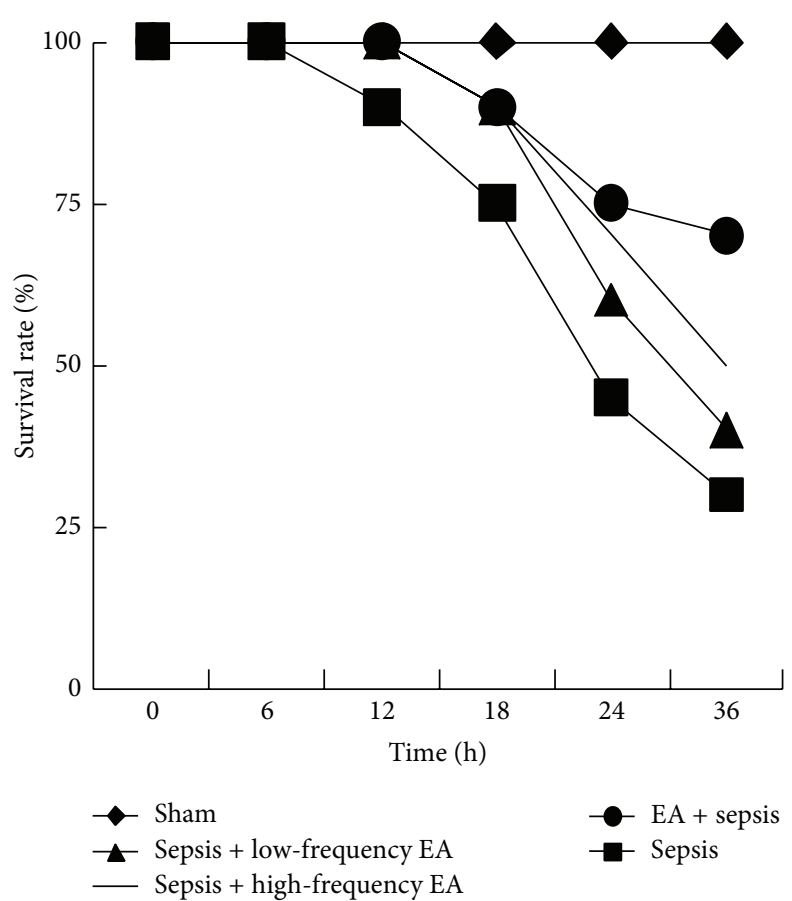

FIGURE 1: EA preconditioning therapy improved the survival rate in a rat model of sepsis induced by CLP. The percentage survival in 72 hours after surgery is shown.

(Borunlaite Sci \& Tech Co., Ltd., Beijing, China) density centrifugation to remove erythrocytes. More than $96 \%$ of the isolated cell population was MC.

For cytofluorometric analyses cells were preincubated in PBS and stained for $30 \mathrm{~min}$ at $4^{\circ} \mathrm{C}$ using saturating concentrations of the following antibodies: CD3-FITC $+\gamma / \delta$ T-PE and CD4-FITC+CD8-PE antibody (Becton, Dickinson and Company, Franklin Lakes, NJ, USA). Cells were analyzed in a Becton-Dickinson LSRII cytometer using FACS Diva software (Becton Dickinson).

\section{Result}

3.1. Survival Study. Survival rate of animals was determined. In sepsis, treatment and prevention groups showed significantly lower survival rate compared to sham group. Although low-frequency EA and high-frequency EA treatment groups had more survival rate than sepsis group (50\% and $37 \%$ versus $26 \%$ ), the difference between sepsis and these groups was not significant. In EA + sepsis group, EA preconditioning therapy improved the survival rate significantly (70\%) (Figure 1).

\subsection{EA at ST36 Ameliorates CLP-Induced Intestinal Injury.} The degree of intestinal injury sepsis was evaluated in CLP rats treated with or without EA at ST36. As shown in Figure 2(a), histological analysis showed that the ileum from sham mice had the normal architecture of the intestinal epithelium and wall, while CLP induced severe edema and sloughing of the villous tips, as well as infiltration of inflammatory cells into the mucosa. Semiquantitative analysis of histological samples of ileum showed that the intestinal injury score in the septic mice was significantly increased compared with that in the sham group. Administration of EA at ST36 significantly decreased CLP-induced intestinal injury (Figure 2(b)).

We also detected the circulating D-Lactose that can be considered an indirect indication of intestinal permeability. As shown in Figure 2(c), the concentration of circulating D-Lactose was increased significantly in the septic mice. Treatment with high-frequency EA and EA pretreatment could significantly inhibit the increase of circulating DLactose induced by CLP.

3.3. Changes of sIgA Content in Intestinal Mucosa Cells. The sIgA concentrations in intestinal mucosa reduced significantly $36 \mathrm{hrs}$ after CLP was developed, while the intestinal mucosal tissue sIgA levels in high-frequency EA group and EA pretreatment group were increased significantly (Figure 3).

3.4. Percentage of CD3+, CD4+, and CD8+ T Lymphocytes in Intestinal Mucosa. As shown in Figure 4, compared with sham group, CD3+, $\gamma / \delta, \mathrm{CD} 4+$, and CD $4+/ \mathrm{CD} 8+\mathrm{T}$ lymphocytes were significantly decreased from $(80.75 \pm 10.24) \%$, $(18.64 \pm 7.73) \%,(24.59 \pm 6.60) \%$, and $(1.89 \pm 0.52)$ to $(38.38 \pm$ $10.90) \%$, $(7.62 \pm 1.79) \%$, $(7.95 \pm 2.95) \%$, and $(0.97 \pm 0.67)$ in the sepsis group. Low-frequency EA could increase the percentage of CD3,$+ \gamma / \delta$, and CD $4+$ T cells as compared with model group. EA preconditioning significantly increased the percentage of $\mathrm{CD} 3+, \gamma / \delta$, and CD $4+\mathrm{T}$ cells and the ratio of 


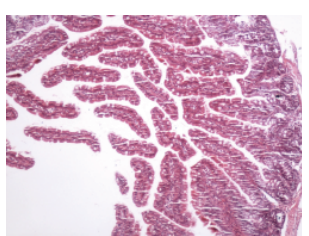

$(\times 100)$

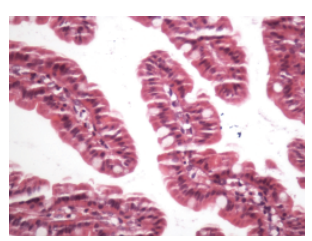

$(\times 400)$

(A)

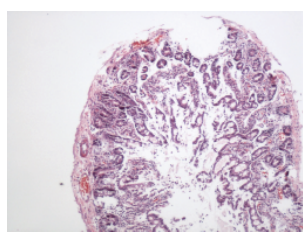

$(\times 100)$

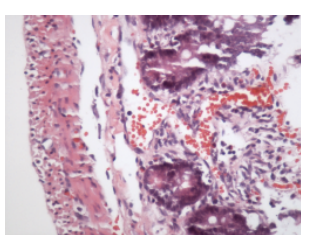

$(\times 400)$

(B)

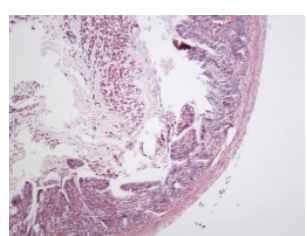

$(\times 100)$

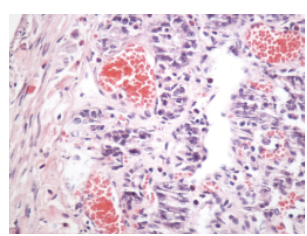

$(\times 400)$

(C)

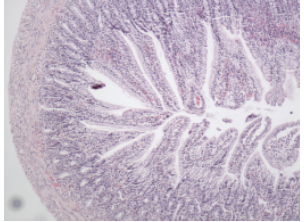

$(\times 100)$

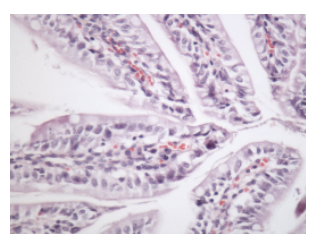

$(\times 400)$

(D)

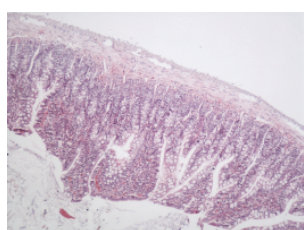

$(\times 100)$

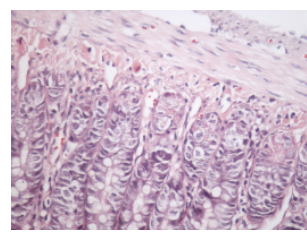

$(\times 400)$

(E)

(a)

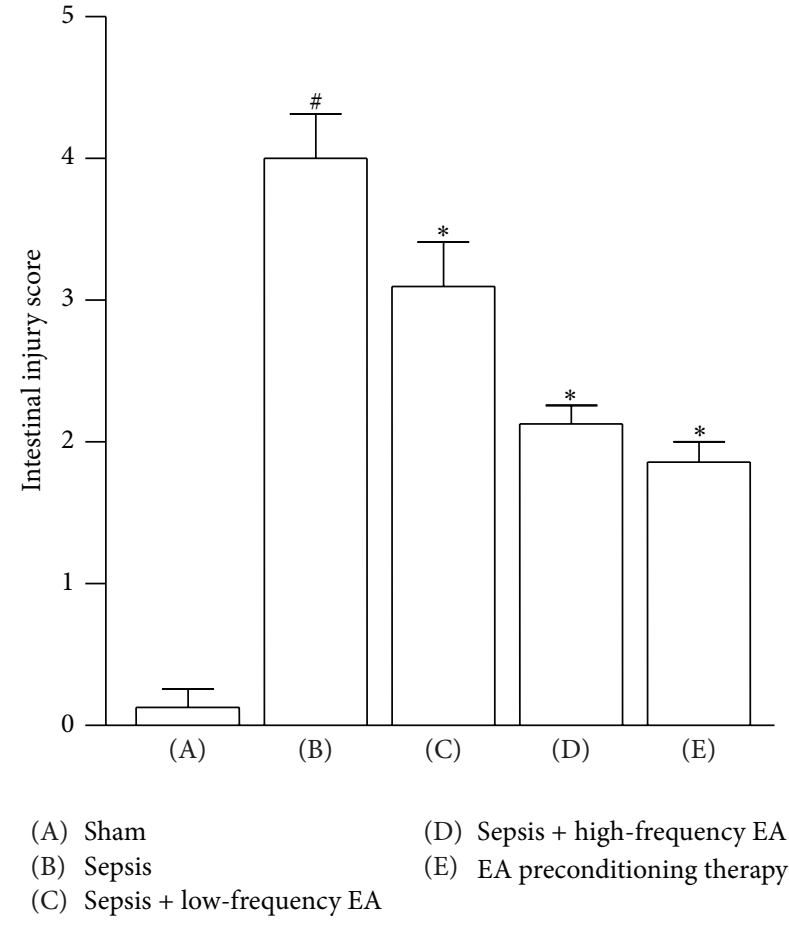

(b)

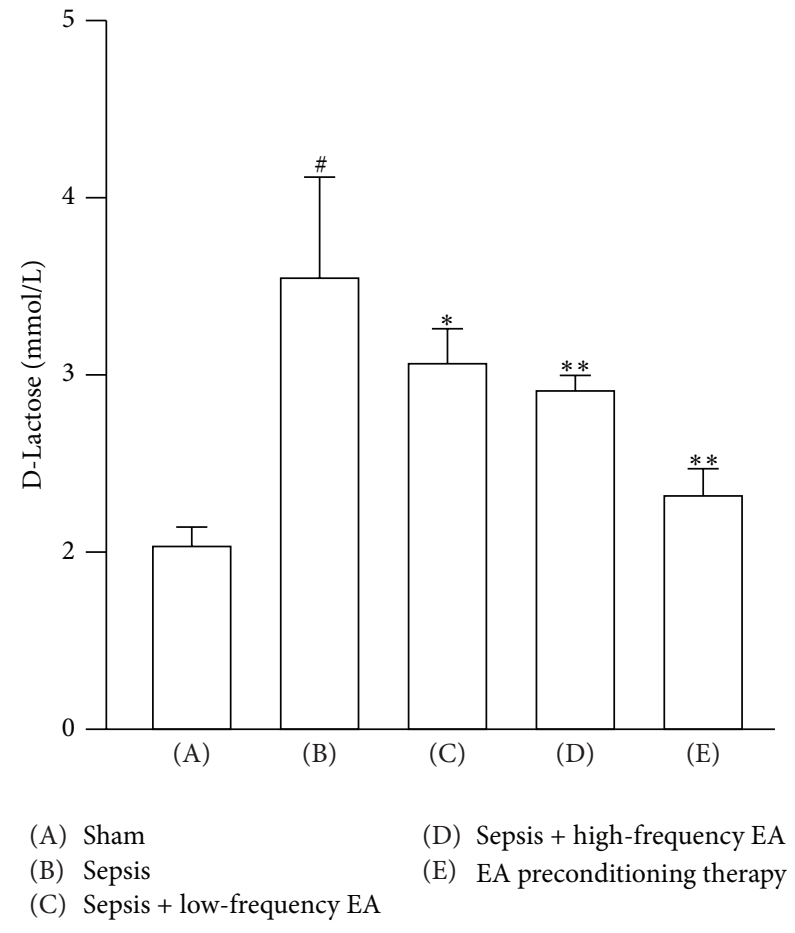

(c)

FIGURE 2: EA at ST36 ameliorates CLP-induced intestinal injury. (a) Ileums were harvested $36 \mathrm{~h}$ after CLP for histopathologic examination using H\&E staining. Representative images from five animals per group were shown. (b) Semiquantitative analysis of histological samples of ileum showed that EA at ST36 significantly decreased CLP-induced intestinal injury. (c) Effects of EA on the concentration of D-Lactose in the serum. Data were presented as means $\pm \operatorname{SD}(n=5)$ and ${ }^{*} p<0.01,{ }^{*} p<0.05$, and ${ }^{* *} p<0.01$ difference with sham or sepsis group.

CD4+/CD8+ T cells to $(77.08 \pm 14.43) \%$, $(20.33 \pm 4.84) \%$, $(20.14 \pm 2.94) \%$, and $(1.43 \pm 0.15)$. However, no difference of the percentage of CD8+ T cells was observed between five groups.

\section{Discussion}

In the present studies, we showed that the intestinal mucosal immune barrier was seriously damaged in a rat model of sepsis induced by CLP. EA preconditioning at ST36 obviously ameliorated CLP-induced intestinal injury and high permeability and exerted protective effects on intestinal mucosal immune barrier; EA preconditioning significantly increased the percentage of $\mathrm{CD} 3+, \gamma / \delta$, and CD $4+\mathrm{T}$ cells and the ratio of $\mathrm{CD} 4+/ \mathrm{CD} 8+\mathrm{T}$ cells and ultimately reduced the mortality of CLP-induced sepsis in rats. Although EA treatments at ST36 with low-frequency and high-frequency could not reduce the mortality of CLP-induced sepsis in rats, these EA treatments could also significantly improve intestinal injury 


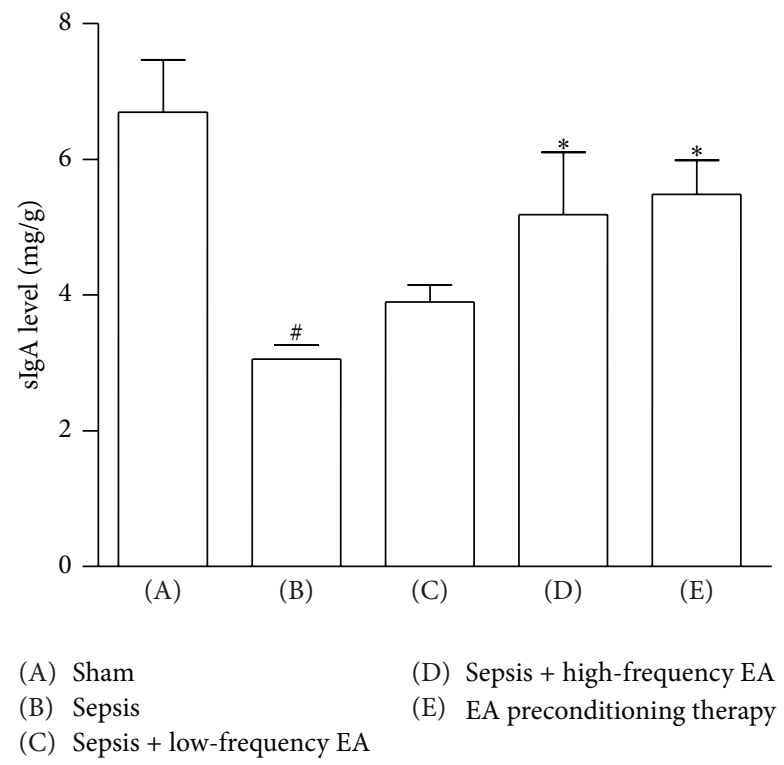

Figure 3: Changes of sIgA content in intestinal mucosa cells. Data were presented as means $\pm \operatorname{SD}(n=5)$ and ${ }^{\#} p<0.01$ and ${ }^{*} p<0.05$ difference with sham or sepsis group.

index in rats with sepsis and obviously protected intestinal mucosal immune barrier.

The gastrointestinal tract is an organ of digestion and absorption. In recent years, the gastrointestinal tract has assumed more importance in the management of the septic patient in the intensive care unit [14-16]. It is now recognized that the small intestine and colon make important contributions to the maintenance of hypermetabolism in sepsis. Owing to the increased intestinal permeability with gut barrier injury, the bacteria and lipopolysaccharide (LPS) can enter the systemic circulation through the portal vein and the mesenteric lymph and result in sepsis and multiple organ dysfunction syndromes (MODS) [17]. Therefore, the intestinal tract is regarded as "initiator" of MODS and supporting general immune function and maintaining the structure and function of the gastrointestinal tract were possible therapeutic strategies for sepsis $[14,18]$.

Based on semiquantitative histological examination and the mucosal damage index, we found that the ileum from the mice of sham group had the normal architecture of the intestinal epithelium and wall, while CLP induced severe edema and sloughing of the villous tips, as well as infiltration of inflammatory cells into the mucosa. The intestinal injury score in the septic mice was significantly increased. Administration of EA at ST36 significantly decreased CLP-induced intestinal injury. It suggested that EA at ST36 improved the restitution and mechanical barriers of intestinal mucosa in CLP-induced sepsis. Moreover, plasma D-lactate, produced by intestinal bacteria, was developed as a biomarker of intestinal high permeability [19]. Here, we showed that the concentration of circulating D-Lactose was increased significantly in the septic mice. Treatment with high-frequency EA and EA pretreatment could significantly inhibit the increase of circulating D-Lactose induced by CLP. It indicated that
EA could protect the intestinal mucosa epithelial cells and maintain the integrity of gut mucosal barrier.

The gut mucosal barrier comprises both immunological and nonimmunological protective components, with the former being divided into local and systemic components and the latter comprising mechanical and chemical barriers. The sIgA content and percentage of T lymphocytes in intestinal mucosa is important to local intestinal mucosal immune barrier [20]. In this study, both of high-frequency EA treatment and EA preconditioning could increase the intestinal mucosal sIgA concentration. Furthermore, EA preconditioning significantly increased the percentage of CD3,$+ \gamma / \delta$, and CD $4+\mathrm{T}$ cells and the ratio of CD4+/CD8+ T cells.

These results supported that EA treatments at ST36 with low-frequency, high-frequency, and preconditioning can protect the intestinal barrier to different degrees, among which EA preconditioning exerts positive effects on immune barrier and eventually decreases the mortality of sepsis.

\section{Conflict of Interests}

The authors declare that there is no conflict of interests regarding the publication of this paper.

\section{Acknowledgments}

This work was supported by Zhejiang Provincial Major Project "Traditional Chinese Medicine in Prevention and Treatment of Major Diseases” (no. 2012ZGG001), Zhejiang Provincial Outstanding Young Talent Program of Traditional Chinese Medicine (no. 2013ZQ013), and Zhejiang Provincial Program for the Cultivation of High-level Innovative Health Talents. 

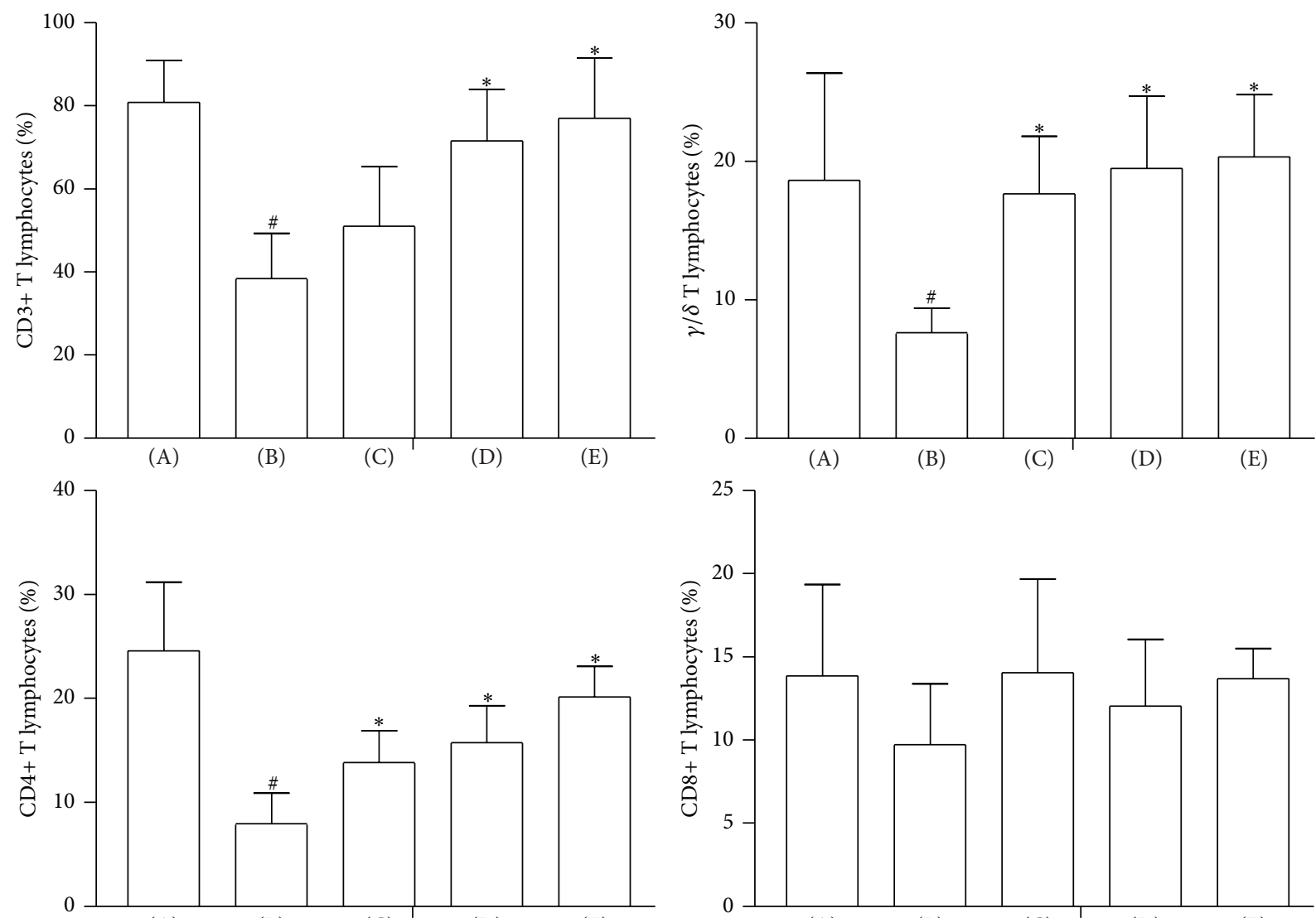

(C)

(A)

(B)

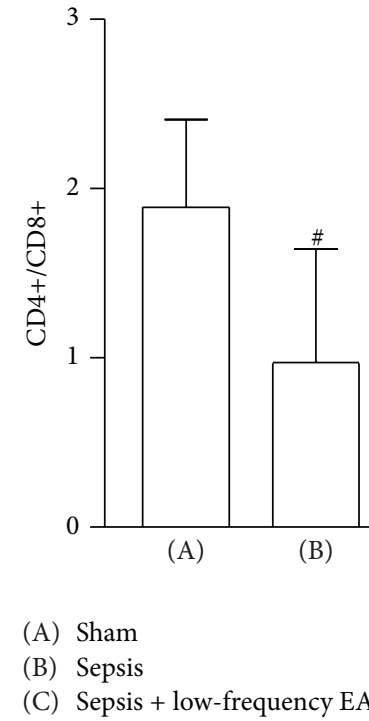

(C)

(D)

(E)

(B)

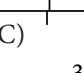

(D)

(E)

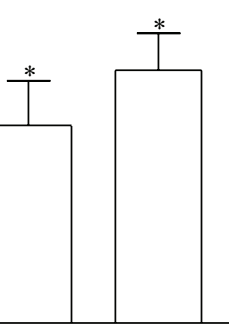

(A)

(C)

(D)

(E)

Figure 4: Effects of EA on percentage of CD3+, CD4+, and CD8+ T lymphocytes in intestinal mucosa. Data were presented as means \pm SD $(n=5)$ and ${ }^{\#} p<0.01$ and ${ }^{*} p<0.05$ difference with sham or sepsis group.

\section{References}

[1] R. P. Dellinger, M. M. Levy, A. Rhodes et al., "Surviving sepsis campaign: international guidelines for management of severe sepsis and septic shock, 2012," Intensive Care Medicine, vol. 39, no. 2, pp. 165-228, 2013.

[2] N. Kissoon, J. A. Carcillo, V. Espinosa et al., "World federation of pediatric intensive care and critical care societies: global sepsis initiative," Pediatric Critical Care Medicine, vol. 12, no. 5, pp. 494-503, 2011.

[3] E. Abraham and M. Singer, "Mechanisms of sepsis-induced organ dysfunction," Critical Care Medicine, vol. 35, no. 10, pp. 2408-2416, 2007.

[4] Global Sepsis Aliance, "World Sepsis Day," 2012, http://www .world-sepsis-day.org. 
[5] J. Cohen, “The immunopathogenesis of sepsis," Nature, vol. 420, no. 6917 , pp. 885-891, 2002.

[6] G. F. Weber and F. K. Swirski, "Immunopathogenesis of abdominal sepsis," Langenbeck's Archives of Surgery, vol. 399, no. 1, pp. $1-9,2014$.

[7] J. C. Marshall, N. V. Christou, and J. L. Meakins, “The gastrointestinal tract: The 'undrained abscess' of multiple organ failure," Annals of Surgery, vol. 218, no. 2, pp. 111-119, 1993.

[8] E. A. Deitch, R. Rutan, and J. P. Waymack, "Trauma, shock, and gut translocation," New Horizons, vol. 4, no. 2, pp. 289-299, 1996.

[9] Z.-J. Zhang, X.-M. Wang, and G. M. McAlonan, "Neural acupuncture unit: a new concept for interpreting effects and mechanisms of acupuncture," Evidence-Based Complementary and Alternative Medicine, vol. 2012, Article ID 429412, 23 pages, 2012.

[10] A. Villegas-Bastida, R. Torres-Rosas, L. A. Arriaga-Pizano, J. Flores-Estrada, A. Gustavo-Acosta, and M. A. Moreno-Eutimio, "Electrical stimulation at the ST36 acupoint protects against sepsis lethality and reduces serum TNF levels through vagus nerve- and catecholamine-dependent mechanisms," EvidenceBased Complementary and Alternative Medicine, vol. 2014, Article ID 451674, 8 pages, 2014.

[11] D. N. Aguiar, M. M. Silva, W. V. Parreira et al., "Electroacupuncture at the ST36 acupoint increases interleukin4 responsiveness in macrophages, generation of alternatively activated macrophages and susceptibility to Leishmania major infection," Chinese Medicine, vol. 7, article 17, 2012.

[12] X.-Y. Suo, Z.-H. Du, H.-S. Wang et al., "The effects of stimulation at acupoint ST36 points against hemorrhagic shock in dogs," The American Journal of Emergency Medicine, vol. 29, no. 9, pp. 1188-1193, 2011.

[13] Y.-K. Yim, H. Lee, K.-E. Hong et al., "Electro-acupuncture at acupoint ST36 reduces inflammation and regulates immune activity in collagen-induced arthritic mice," Evidence-Based Complementary and Alternative Medicine, vol. 4, no. 1, pp. 5157, 2007.

[14] B. J. Rowlands, C. V. Soong, and K. R. Gardiner, "The gastrointestinal tract as a barrier in sepsis," British Medical Bulletin, vol. 55, no. 1, pp. 196-211, 1999.

[15] M. S. Penn and M. Kamath, "Novel mechanisms for maintaining endothelial barrier function in sepsis," Circulation, vol. 126, no. 23, pp. 2677-2679, 2012.

[16] E. A. Deitch, "Gut-origin sepsis: evolution of a concept," Surgeon, vol. 10, no. 6, pp. 350-356, 2012.

[17] J. MacFie, C. O’Boyle, C. J. Mitchell, P. M. Buckley, D. Johnstone, and P. Sudworth, "Gut origin of sepsis: a prospective study investigating associations between bacterial translocation, gastric microflora, and septic morbidity," Gut, vol. 45, no. 2, pp. 223-228, 1999.

[18] D.-C. Chen and L. Wang, "Mechanisms of therapeutic effects of rhubarb on gut origin sepsis," Chinese Journal of Traumatology, vol. 12, no. 6, pp. 365-369, 2009.

[19] M. D. Welch, "Lactate as an early marker of intestinal ischaemia after raptured abdominal aortic aneurysm repair," British Journal of Surgery, vol. 86, no. 5, pp. 712-714, 1998.

[20] R. T. Maxson, D. D. Johnson, R. J. Jackson, and S. D. Smith, “The protective role of enteral IgA supplementation in neonatal gutorigin sepsis," Annals of the New York Academy of Sciences, vol. 778, pp. 405-407, 1996. 


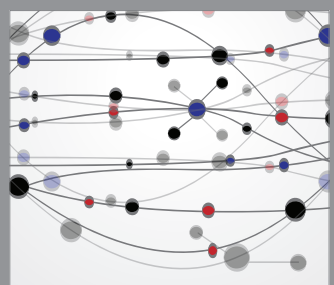

The Scientific World Journal
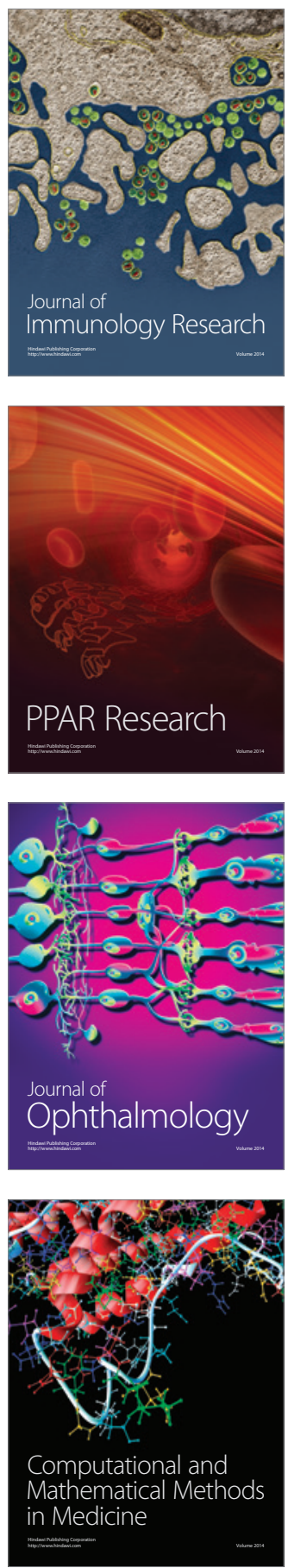

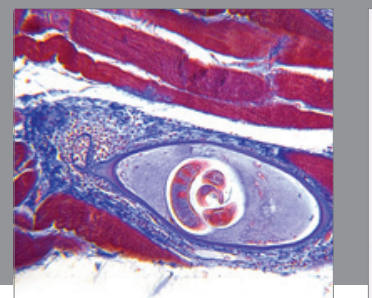

Gastroenterology

Research and Practice
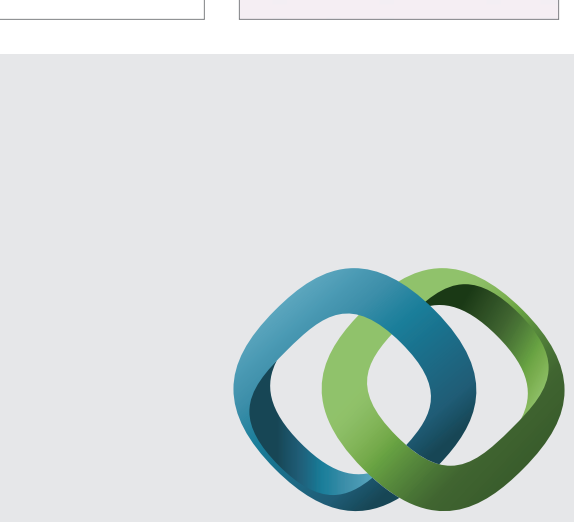

\section{Hindawi}

Submit your manuscripts at

http://www.hindawi.com
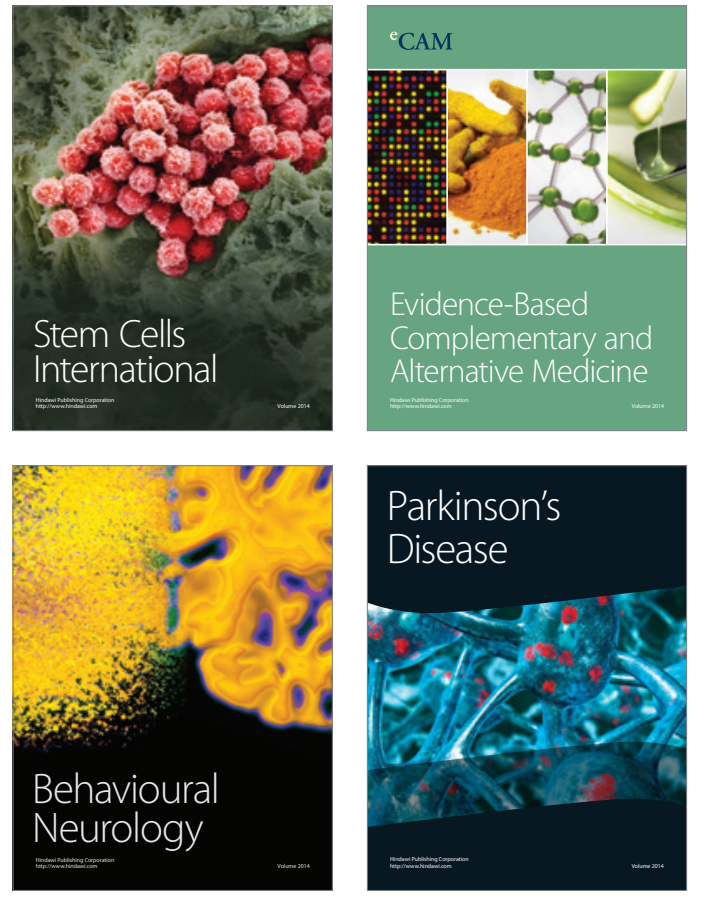
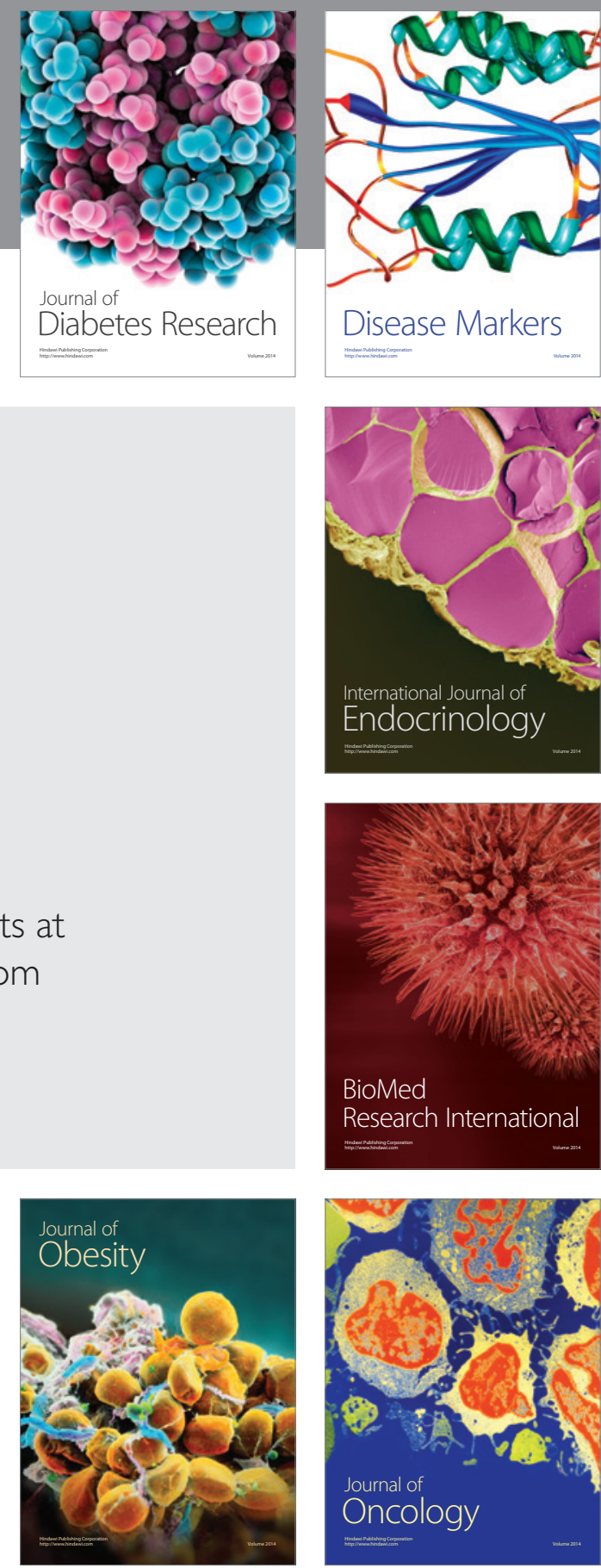

Disease Markers
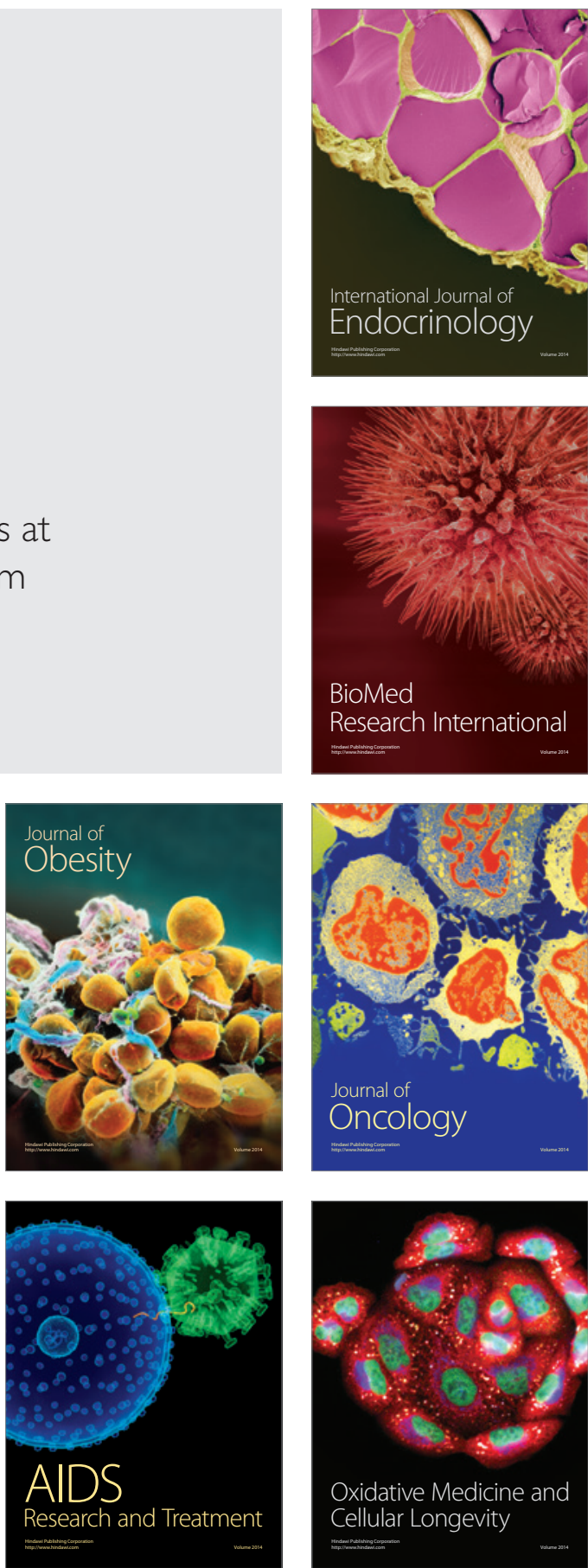